\title{
Synthèse
}

\section{Les insectes ravageurs importants de la pomme de terre au Canada}

\author{
Christine Noronha ${ }^{1}$ \\ Robert S. Vernon ${ }^{2}$ \\ Charles Vincent ${ }^{3}$ \\ ${ }^{1}$ Agriculture and Agri-Food Canada, \\ Crops and Livestock Research Centre, \\ Charlottetown, P. E. I., \\ C1A 4N6, \\ Canada \\ <noronhac@agr.gc.ca> \\ 2 Pacific Agri-food Research Station, \\ Agriculture and Agri-Food Canada, \\ Agassiz, B.C \\ Canada VOM 1 AO \\ ${ }^{3}$ Centre de recherche et de développement \\ en horticulture, agriculture et agro-alimentaire \\ Canada, \\ 430, boulevard Gouin \\ Saint-Jean-sur-Richelieu, Qc, \\ J3B 3E6 \\ Saint-Jean-sur-Richelieu, \\ Canada \\ <vincentch@agr.gc.ca>
}

\begin{abstract}
Résumé
La pomme de terre contribue pour environ $63 \%$ au chiffre d'affaire du marché des légumes canadiens. Comme la plupart des plantes cultivées, les rendements sont entravés par plusieurs espèces d'insectes ravageurs tels que le doryphore de la pomme de terre, la pyrale du maïs et les taupins. L'absence de mesures antiparasitaires peut conduire à des pertes majeures en rendement. Les producteurs canadiens ont recours à des approches de lutte intégrée en utilisant plusieurs techniques pour gérer les insectes ravageurs et maintenir leurs populations en deçà de leurs seuils économiques. Ces techniques comprennent le dépistage à l'aide de pièges à phéromone ou appâtés, l'utilisation de modèles de degrés-jours pour estimer l'émergence des adultes, le dépistage en champ pour l'estimation des populations, l'utilisation de seuils économiques pour l'application plus précise d'insecticides et des méthodes de lutte biologique, physique et culturale. L'objectif de plusieurs projets de recherche au Canada est de fournir des connaissances plus approfondies sur ces insectes. L'objectif poursuivi par les producteurs et les chercheurs est une réduction des quantités d'insecticides utilisées pour gérer les populations d'insectes ravageurs de la pomme de terre.
\end{abstract}

Mots clés : insecte nuisible, lutte intégrée, Solanum tuberosum

Thèmes : pathologie, productions végétales

\section{Abstract Key pests of potatoes in Canada}

The potato crop is estimated to contribute $63 \%$ to the vegetable revenue in Canada. Like most agricultural crops, the potato crop yield is compromised by attacks of several insect pests during the growing season. Pests such as the Colorado potato beetle, the European corn borer, wireworms and tuber flea beetle can cause serious problems for potato production. Lack of control applications can result in major economic losses to the producer. In Canada, growers employ an Integrated Pest Management approach by using several techniques to manage and keep pest populations below economic thresholds in the potato crop. Some of the techniques used are: monitoring populations with pheromone and bait traps, use of degree days to estimate emergence, scouting fields to determine population levels, use of economic thresholds for a more precise application of insecticides and biological, cultural and mechanical control techniques. The objective of several research projects in Canada is to develop and refine control techniques and obtain a better understanding of the pests. The main objective for both the growers and the researchers is to reduce the amount of pesticides needed to control these insect pests in potatoes, while maintaining economic benefits for the producers.

Keywords: insect pests, integrated control, Solanum tuberosum

Subjects: pathology, vegetal productions

a pomme de terre génère $63 \%$ du chiffre d'affaire du marché des légumes cultivés au Canada. En 2006, les surfaces cultivées étaient de
161706 ha, la production étant effectuée à l'Île-du-Prince-Édouard (49 256 ha), au Manitoba (32983 ha), au NouveauBrunswick (23 473 ha), en Alberta 
(22 056 ha), au Québec (18 980 ha) et en Ontario (14976 ha).

Chaque année, de nombreuses espèces d'insectes attaquent la pomme de terre, les principales espèces étant le doryphore de la pomme de terre (Colorado potato beetle) Leptinotarsa decemlineata (Coleoptera: Chrysomelidae), la pyrale du maïs (European corn borer) Ostrinia nubilalis (Lepidoptera: Crambidae), quatre espèces de pucerons (Homoptera: Aphididae) et les taupins (wireworm) [Coleoptera: Elateridae]. Ces insectes constituent des préoccupations majeures pour les industries canadiennes et américaines de la pomme de terre. Dans les provinces maritimes, la plupart des producteurs ont recours à des experts en dépistage pour connaître les niveaux de populations de ces ravageurs dans leurs champs. Des mesures phytosanitaires sont prises lorsque les niveaux de populations dépassent les seuils économiques. Le cas des pucerons étant traité par Le Roux et al. (2008), nous ne développerons pas la problématique inhérente à ces ravageurs. Mentionnons cependant que des projets de recherche sont en cours au Canada en partenariat avec Agriculture et agro-alimentaire Canada (AAC) [Frédéricton et Saint-Jean-sur-Richelieu], l'université du Québec à Montréal, l'université de Picardie Jules-Verne (Amiens, France) et des groupements de producteurs des deux côtés de l'Atlantique. Ces projets visent à développer des cultivars résistants aux pucerons.

\section{Doryphore de la pomme de terre}

Le doryphore de la pomme de terre est l'insecte le plus important dans les cultures de pomme de terre en Amérique du Nord. On le retrouve dans toutes les régions où l'on cultive de la pomme de terre en Amérique du Nord. En l'absence de méthodes de lutte, les larves et les adultes peuvent défolier complètement les plants, ce qui réduit la capacité photosynthétique des plantes et se traduit par des pertes de rendement.

L'application d'insecticides constitue la principale méthode de lutte en Amérique du Nord : en conséquence, cet insecte a développé des résistances à la plupart des insecticides homologués (Roush et al., 1990 ; Boiteau et al., 1987 ; Boiteau, 1988 ; Harris et Svec, 1981 ; Stewart et al., 1997). En cas d'échec de lutte en raison du développement de résistances, des pertes importantes peuvent survenir. À l'heure actuelle, les populations de doryphores sont contrôlées avec des insecticides de nouvelle génération, comme l'imidaclopride. Toutefois, le développement potentiel de résistances à l'encontre de ces nouveaux pesticides est une préoccupation constante pour les producteurs.

A la fin du quatrième stade, les larves se laissent choir sur le sol, où elles s'enfouissent pour se nymphoser. Trois semaines plus tard, les adultes émergent et commencent à s'alimenter, voire à pondre dans les régions où il y a deux générations. Au Canada et dans les autres régions froides de son aire de distribution géographique, les nouvelles générations d'adultes émergent à la mi-saison, en juillet.

La diapause est induite en réponse à une photophase et à des températures à la baisse en interaction avec la maturité des plants. Au lieu de conduire à la reproduction, elle induit une prise de nourriture intense nécessaire au stockage de réserves (Hoy et al., 1996 ; Noronha et Cloutier, 1999,2007). Noronha et Cloutier (2007) ont démontré que la température influence le taux et la durée de prise de nourriture des adultes qui se nourrissent préférentiellement de feuillage âgé. L'exposition à une combinaison de feuilles âgées et de températures élevées diminue le taux de survie lors de la diapause. Les adultes quittent le plant et marchent ou volent vers les rangs en périphérie des champs ou les boisés avoisinants (Boiteau, 2001 ; Noronha et Cloutier, 1999; Voss et Ferro, 1990 ; Noronha et al., 2002). Une fois en périphérie, les adultes s'enfouissent dans le sol à 10-30 cm et entrent en diapause (Minder, 1976 ; Noronha et Cloutier, 1998 ; Ushatinskaya, 1978 ; Weber et Ferro, 1993). La profondeur à laquelle les adultes diapausent est influencée par la température, l'humidité et la densité du sol (Noronha et Cloutier, 1998). Lors de la phase hivernale, la tolérance au froid des adultes se situe à une moyenne de -7 à $-11^{\circ} \mathrm{C}$ (Boiteau et Coleman, 1996 ; Lee et al., 1994). Toutefois, la neige a tendance à s'accumuler dans les haies, ce qui isole les sols et les maintient à des températures supérieures à $0{ }^{\circ} \mathrm{C}$, à des profondeurs de 5 à $20 \mathrm{~cm}$, où la majorité des adultes diapausent. Dans la plupart des régions canadiennes et dans les régions septentrionales américaines, le doryphore a une génération par an.
Quoique les insecticides utilisés actuellement permettent un contrôle efficace du doryphore en Amérique du Nord, sa capacité d'adaptation aux insecticides est telle que l'on effectue un suivi constant des populations pour déterminer le développement de résistance et prévenir des dommages importants. On recommande aux producteurs de pratiquer la rotation des cultures, le dépistage au champ pour déterminer les niveaux de populations et de ne réaliser des traitements insecticides que lorsque les seuils économiques sont atteints. On leur recommande également d'effectuer une rotation des classes d'insecticides homologués au cours de la saison pour retarder l'apparition de populations résistantes (Noronha et al., 2001). La mise en ouvre de cette recommandation dépend du marché ciblé. Ainsi, des producteurs canadiens qui voudraient vendre leurs pommes de terre aux État-Unis doivent se conformer aux réglementations de ce pays, notamment en ce qui concerne l'utilisation et les résidus des certains pesticides. Ces contraintes sont par ailleurs également mises en force par les transformateurs canadiens afin de respecter le cadre réglementaire de leurs clients américains. Il existe des moyens alternatifs de lutte, parmi lesquels la lutte thermique (Duchesne et al., 2000), les barrières physiques (Boiteau et al., 1994 ; Boiteau et Vernon, 2000) et les méthodes pneumatiques (Vincent et Boiteau, 2000). Des recherches ayant pour objectif de développer des cultivars résistants (ex. :Pelletier et al., 2001 ; Pelletier et Clark, 2004) et des agents de lutte biologique, tels que Beauvaria bassiana et Podisus maculiventris, sont en cours.

\section{Pyrale du maïs}

La pyrale du maïs, O. nubilalis, qui se nourrit sur plus de 200 espèces de plantes, est considérée comme un ravageur important du maïs. Cet insecte occasionnellement trouvé sur la pomme de terre était auparavant considéré comme un ravageur secondaire. Depuis 1987, les chercheurs des Provinces Maritimes ont observé une augmentation constante des dommages causés à la pomme de terre par ce ravageur. La pyrale du maïs est dorénavant considérée comme un ravageur des pommes de terre cultivées à l'Île-du-Prince-Édouard, au NouveauBrunswick et en Nouvelle-Écosse. Elle 
cause occasionnellement des dégâts dans les cultures au Québec, en Ontario et au Manitoba. En 2004, les niveaux d'infestations (basés sur le nombre d'œufs par plant) variaient de 10 à $90 \%$, avec des moyennes de cinq tunnels et trois larves par tige dans certains champs. La pyrale du maïs a une génération dans la région Atlantique du Canada. Les papillons émergent du début à la mi-juin et pondent sur les feuilles et les tiges du tiers inférieur du plant (Stewart, 1992). Les larves éclosent au bout d'une semaine et se forent un passage dans les tiges. Elles causent leurs dommages principaux lorsqu'elles forent des tunnels dans les tissus constitutifs des tiges, restreignant ainsi le flux de nutriments vers les tubercules et les structures en croissance. Selon des études menées aux États-unis sur des variétés hâtives, les baisses de rendements des pommes de terre de grade US-1 sont négligeables (Kennedy, 1983 ; Kennedy et Anderson, 1980 ; Nault et Kennedy, 1996a). Toutefois, Stewart (1992) rapporte des baisses de rendements chez les variétés tardives de l'îledu-Prince-Édouard, chez les plants ayant plus de 1,6 larve par tige. Les trous et les tunnels affaiblissent les tiges, ce qui les rend plus susceptibles à la cassure par les vents et aux stress hydriques, et offre des portes d'entrées aux pathogènes comme la jambe noire, Erwinia carotovora (var. atroseptica). Les producteurs et les dépisteurs utilisent des pièges et des modèles degrés-jours pour prédire le début des pontes (Dornan et Stewart, 1995; Stewart, 1994).

Nault et Kennedy (1996b) ont trouvé que des traitements insecticides, effectués selon des comptages de masses d'œufs, donnent des résultats plus fiables parce que les trous effectués en début de saison sont minuscules et peuvent être difficilement repérés. Les insecticides constituent la principale méthode de lutte, et les applications doivent être synchronisées précisément pour atteindre les larves néonates, car celles-ci sont protégées une fois dans la tige. La durée pendant laquelle la larve néonate est exposée à l'extérieur de la tige est méconnue. Les producteurs utilisent une fenêtre de traitement de deux jours suivant le pic d'éclosion des œufs comme époque optimale de traitement. Les traitements insecticides sont souvent retardés en raison de mauvaises conditions météorologiques, ce qui cause des baisses de rendements. $\mathrm{Au}$ pic de ponte des oufs, les producteurs effectuent souvent deux traitements insecticides à une semaine d'intervalle. Certains insecticides à risques réduits comme le spinosad, le novaluron et l'indoxacarb sont des larvicides efficaces. Ces insecticides réduisent les dommages lorsqu'ils sont appliqués au stade des têtes noires des larves. Le novaluron est aussi efficace lorsqu'il est appliqué sur les œufs en champ (Boiteau et Noronha, 2007 ; Noronha et Carragher, 2006). Ces insecticides, qui offrent une flexibilité accrue lors de périodes de météorologie inclémente, permettent aux producteurs d'appliquer des insecticides seulement lorsque les seuils sont dépassés. Les producteurs utilisent comme seuil provisoire de traitement insecticide un seuil d'infestation de $20 \%$ des plantes, lequel est basé sur le dénombrement de masses d'œufs. Les études sur la réduction des rendements suite à des infestations ont donné des résultats variables: certaines indiquent des pertes significatives seulement lorsque les infestions sont supérieures à 70 \%. Dau-Schmidt et Noronha (données non publiées) ont étudié les effets de cinq niveaux d'infestations sur les rendements de deux variétés (Russet Burbank et Shepody). Les résultats préliminaires indiquent des baisses totales de rendements significatives supérieures à $50 \%$. Il y a une augmentation significative des calibres chez les petites pommes de terre et une diminution significative des calibres chez les grosses pommes de terre. Cela indique que le plant pourrait compenser lorsqu'il est stressé par les larves de pyrale du maïs et que ce phénomène pourrait ne pas être apparent si l'on considère les rendements totaux. En conséquence, le rendement total n'est peut-être pas un prédicteur fiable des pertes de rendements causés par la pyrale. Une augmentation de la proportion de petits tubercules signifie une réduction des prix de vente. Ces effets n'ont pas été observés chez la variété hâtive Shepody. La relation mathématique entre le nombre de trous et les dommages peut être calculée comme suit :

(nombre de tunnels $+0,5)=0,84( \pm 0,03)$

$\sqrt{(\text { nombre de trous }+0,5)}+0,81( \pm 0,34)$

Les nymphes de la pyrale du maïs passent l'hiver dans les tiges laissées au champ. En général, les procédures de récolte impliquent l'enlèvement des tubercules avec les tiges séchées de la surface du sol. Les papillons émergent des tiges laissées au sol, le printemps suivant. Une méthode physique de gestion de ce ravageur consiste à écraser les pyrales avec un appareil attaché à une récolteuse tractée (Noronha, 2006). Les tubercules sont d'abord récoltés et les tiges sont écrasées par la suite. L'efficacité de cet appareil est de 85 à $90 \%$.

\section{Taupins}

Les vers fils de fer (click beetles) (Coleoptera: Elateridae) sont devenus un des groupes de ravageurs polyphages les plus importants au monde. Au Canada, plusieurs espèces indigènes et introduites, ayant des cycles vitaux et des niches écologiques uniques, posent des défis majeurs en matière de phytoprotection. En Colombie-Britannique, deux espèces introduites d'Europe, Agriotes obscurus L. (dusky wireworm) et $A$. lineatus L. (lined click beetle) (Wilkinson, 1963), sont des ravageurs importants. Dans les Provinces Maritimes, ces deux espèces et une autre espèce européenne, A. sputator L., sont les espèces dominantes (Eidt, 1953).

Au Manitoba, en Saskatchewan et en Alberta, les principales espèces sont Limonius canus (LeConte) (Pacific coast wireworm), L. californicus (Mann) (sugarbeet wireworm), Ctenicera destructor (Brown) (Prairie grain wireworm), A. mancus (wheat wireworm) et d'autres espèces. En Ontario et au Québec, les principales espèces sont A. mancus, L. agonis (Say) [eastern field wireworm] et un complexe d'espèces de Melanotus spp. (e.g.: Melanotus communis) (Glen et al., 1943).

En raison de leur mode de vie souterrain, les taupins peuvent causer des pertes catastrophiques en rendements (chez les céréales, le maïs, les légumes, les petits fruits et les plantes ornementales) et des pertes de qualités (chez la carotte, la fraise et la pomme de terre) (Wilkinson, 1963; Vernon, 2005). Les dommages de taupins sont généralement plus importants dans les champs qui étaient auparavant des pâturages ou des champs de céréales, car ce sont leurs plantes hôtes préférées pour la ponte (Wilkinson, 1963). Après l'enlèvement de ces cultures, les taupins qui y restent se nourrissent alors de cultures à haute valeur ajoutée, cultivées en rotation à proximité. En raison de leur cycle vital long (deux à six ans selon les espèces), les taupins peuvent causer des dommages pour plusieurs années.

Depuis la Seconde Guerre mondiale, les méthodes de lutte pratiquées de par le 
monde reposent sur l'utilisation d'insecticides organochlorés, organophosphorés et de carbamates appliqués au sol ou aux semences (ex. : céréales, maïs) (Parker et Howard, 2001). Une seule application d'insecticides organochlorés persistants tels que l'aldrine ou l'heptachlor pouvait contrôler les populations de taupins pour une durée de neuf ans (Wilkinson et al., 1964). Jusqu'en 2003, l'insecticide organochloré, lindane, (un des moins persistant de ce groupe) était utilisé pour réduire les dommages et les populations de taupins dans les provinces des Prairies (Toba et al., 1988). En culture de pomme de terre, l'utilisation d'insecticides organophosphorés et de carbamates, comportant des risques plus élevés mais des propriétés résiduelles moindres que les organochlorés, a assuré des niveaux de contrôle adéquat, suite à l'interdiction de ces derniers dans les années 1970. Au Canada, la plupart des insecticides ont été interdits dans toutes les cultures : seul le phorate (Thimet $15 \mathrm{G}$ ) est homologué pour le contrôle des taupins en pomme de terre. En conséquence, l'importance des dégâts dans plusieurs cultures sensibles, notamment le blé et le maïs, a considérablement augmenté et le contrôle des taupins avec le phorate est devenu erratique.

En raison de l'accroissement des problèmes posés par les taupins dans plusieurs cultures économiquement importantes du Canada et de la paucité d'insecticides homologués relativement aux États-Unis, le développement de stratégies alternatives est récemment devenu une priorité pour AAC. Au cours de la décade passée, les priorités de recherches concernant la gestion des taupins étaient :

- des recherches fondamentales sur leur biologie et leur écologie ;

- le développement de programmes de dépistage ;

- le développement de méthodes de lutte.

\section{Recherches fondamentales sur la biologie et l'écologie des taupins}

Au Canada, des inventaires ont été réalisés dans les régions productrices pour déterminer quelles espèces de taupins sont les plus importantes. En collaboration avec Miklos Toth (Institut de protection des plantes, Budapest, Hongrie), des pièges à phéromones ont été développés à AAC-Agassiz (Colombie-Britannique) pour dépister les taupins européens
A. lineatus, A. obscurus et A. sputator. Ces pièges, qui attirent les adultes mâles, ont été utilisés lors d'inventaires effectués en Amérique du Nord (Vernon et al., 2001) et dans des programmes de lutte intégrée en fraisières. Ils ont permis de déterminer que A.sputator est maintenant l'espèce de taupin la plus importante à l'île-du-Prince-Édouard et en NouvelleÉcosse (Vernon et Noronha, données non publiées). En Colombie-Britannique, à l'île-du-Prince-Édouard et en NouvelleÉcosse, les espèces exotiques sont bien établies et ont déplacé les espèces indigènes en abondance et en importance économique.

Jusqu'à récemment, les chercheurs qui étudient les taupins d'origine européenne au Canada croyaient que les adultes $A$. lineatus et $A$. obscurus étaient incapables de voler (Eidt, 1953; Wilkinson, 1963). Cela est contraire à ce qui a été observé en Europe et a des implications sur la dispersion et la gestion de ces insectes avec certaines techniques comme le piégeage de masse ou d'exclusion. En 2002, les mâles et les femelles de ces espèces ont été observés plusieurs fois en vol en Colombie-Britannique (Crozier et al., 2003). En laboratoire, il a été démontré que le vol pouvait être induit à $24^{\circ} \mathrm{C}$. Lors d'études de capturesrecaptures, $A$. lineatus volait plus souvent que $A$. obscurus lors de lâchers à des températures élevées. Des études sur la vitesse et la direction du vol dans et entre les habitats sont en cours en ColombieBritannique ( $A$. obscurus) et à l'Île-duPrince-Édouard (A. sputator).

\section{Développement de programme de dépistage}

Plusieurs techniques ont été développées pour échantillonner les larves de taupins dans le sol, mais elles sont généralement dispendieuses et demandent beaucoup de temps aux producteurs. De plus, elles livrent des résultats variables pour l'estimation des populations de taupins et les risques encourus. En conséquence, le dépistage ne constitue pas un outil fiable utilisé dans la lutte des taupins en pomme de terre et autres cultures au Canada. Les pièges développés pour le dépistage d'A. obscurus et d'A. lineatus ont été évalués pour déterminer si les captures d'adultes permettent une prédiction fiable des risques de dommages en champs. Pour répondre à cette question, des pièges à phéromone pour capturer les adultes et de pièges souterrains pour les lar- ves ont été déployés dans plus de 50 champs en Colombie-Britannique pour évaluer l'abondance relative et la distribution spatiale des larves et des adultes $A$. lineatus et $A$. obscurus. Les résultats indiquent que les captures d'adultes dans les pièges à phéromone sont corrélées $80 \%$ du temps avec les estimations de populations larvaires avec les leurres. Une méthode d'évaluation des risques posés par A. obscurus et A. lineatus consiste à installer cinq pièges à phéromone et cinq pièges appâtés (R. Vernon, non publié).

\section{Développement de méthodes de lutte}

Depuis 2000, les chercheurs de AAC ont fait des progrès considérables pour mettre au point des méthodes en laboratoire et en champ dans le but de développer des méthodes de lutte comportant des insecticides à faibles risques. De 2004 à 2007, les effets à long terme de plusieurs insecticides appartenant à six classes ont été déterminés sur cinq espèces canadiennes, notamment A.lineatus, A. obscurus et A. sputator. Ces études ont amené des informations cruciales concernant la toxicité relative et les effets sublétaux d'insecticides de nouvelle génération envers les taupins. Les néonicotinoïdes (soit l'imidaclopride, le clothianidin et le thiaméthoxam), qui sont des candidats sérieux pour la lutte contre les taupins de par le monde, intoxiquent de façon réversible (plutôt que de tuer) les taupins aux doses homologuées (Vernon et al., 2005 ; Vernon et al., 2008 ; van Herk et al., 2008). Lors de plusieurs bio-essais en laboratoire, l'intoxication à long terme a été démontrée. On a également démontré le retour à un état physiologique normal suite à une exposition à du blé et des pommes de terre traitées avec des néonicotinoïdes en champ à AAC-Agassiz. Vernon, Tolman, Mackenzie et Noronha (rapports non publiés) ont démontré que l'efficacité des néonicotinoïdes à réduire les dommages causés par les taupins à la pomme de terre varie énormément entre les espèces de taupins à travers le Canada. Il y a donc peu de chances que ces insecticides aient une efficacité semblable à celle du phorate, qui est présentement homologué. Toutefois, certains néonicotinoïdes (ex. : le clothianidin et le thiaméthoxam), qui ont des effets sur le doryphore de la pomme de terre et plusieurs espèces d'altises, font l'objet de tractations pour qu'ils soient homologués pour la lutte 
contre les taupins qui attaquent les pommes de terre au Canada.

Le laboratoire de AAC-Saskatoon a été le premier à démontrer l'attraction qu'exerce le $\mathrm{CO}_{2}$ issu de grains germés envers les taupins (Doane et al., 1975). À partir de ces résultats, AAC-Agassiz a développé des méthodes de laboratoires et des modèles pour étudier l'attractivité, la répulsion et la toxicité en conditions de champs et de sol simulées. Ces travaux ont démontré que le tefluthrin, un pyrèthre homologué pour la lutte contre les taupins au Canada, est un répulsif pour toutes les espèces de taupins ayant une incidence économique au Canada (van Herk et Vernon, 2007).

En ce qui a trait à la lutte biologique, des souches uniques du fongi Metarhiziu$m$ anisopliae Metchnikoff (Kabaluk et al., 2005 ; Inglis et al., 2008), isolées à partir d'A. obscurus à Agassiz, sont actuellement évaluées au laboratoire et au champ en cultures de maïs et de pomme de terre. En conditions de laboratoire, Kabaluk et al. (2005) rapportent une bonne efficacité de certains isolats de M. anisopliae envers les larves de C. pruinina (Horn) et envers les larves et les adultes d'A. obscurus et $A$.lineatus. Des applications en champs de $M$. anisopliae ont également causé une réduction des dommages infligés aux tubercules par A. obscurus (Kabaluk et al., 2005). Kabaluk et al. (2007) ont démontré que des concentrations supérieures à $4 \times 10^{6}$ conidies par centimètre cube infectent et tuent les larves d'A. obscurus. En laboratoire, Ericsson et al., (2007) ont démontré que l'insecticide spinosad agit de façon synergique avec $M$. anisopliae, ce qui augmente les mortalités larvaires d'A. obscurus et $A$. lineatus et réduit leurs activités de prise alimentaire. Des études en champs de maïs et de pomme de terre sont actuellement en cours à AACAgassiz, avec M. anisopliae avec ou sans spinosad.

Concernant les méthodes culturales, des études menées à AAC-Agassiz ont démontré qu'un contrôle des taupins peut être fait en plantant des culturespièges de blés traitées avec un insecticide, le Vitavax ${ }^{\circledR}$ dual, qui contient du lindane (Vernon, 2005). Ces culturespièges peuvent être plantées avant l'établissement de cultures sensibles comme la pomme de terre et le fraisier ou peuvent être cultivées de façon alternative en vue d'attirer et de tuer les taupins sans avoir à traiter la culture principale (pomme de terre ou fraisier). De 1998 à
2003, les producteurs de la ColombieBritannique avaient adopté de telles stratégies, mais ils ont cessé ces pratiques suite au retrait du lindane au Canada, en 2003. AAC-Agassiz effectue actuellement des travaux pour trouver un insecticide à faible impact environnemental qui remplacera le lindane. L'inondation des champs est utilisée par certains producteurs de pomme de terre "biologique " pour réduire les populations de taupins l'année précédant la plantation. Lors d'essais préliminaires en laboratoire, la mortalité survient plus rapidement dans des sols à haute salinité et température : le temps requis pour tuer $90 \%$ de la population était d'environ neuf jours à entre 10 et $20^{\circ} \mathrm{C}$, ce qui suggère que le meilleur temps pour inonder un champ du sud-ouest de la Colombie-Britannique est de mai à septembre (van Herk et Vernon, 2006).

En ce qui concerne la lutte sémiochimique, des études effectuées au cours de deux années dans le sud-ouest de la Colombie-Britannique suggèrent que des pièges à phéromones pourraient être utilisés pour capturer les mâles d'A. obscurus en champs de graminées. Toutefois, le coût d'un tel programme de piégeage en masse est pour l'instant prohibitif. Cette approche est moins efficace pour A. lineatus.

Lors d'essais préliminaires de 2000 à 2003 à AAC-Agassiz, les pièges à phéromones ont été utilisés pour cartographier dans le temps la distribution d'A.lineatus et $A$. obscurus en fraisières et en pâturages. Ces inventaires ont démontré que les populations sont décimées par certains traitements d'organophosphorés et de pyrèthres synthétiques, et que la recolonisation des surfaces traitées à partir des populations réservoirs est graduelle. Aux Pays-Bas, les pièges à phéromones ont été récemment utilisés pour synchroniser les traitements insecticides en champs céréaliers et en pâturages en vue d'éliminer les adultes avant qu'ils ne pondent (Ester et van Rozen, 2005). Ce programme, qui est adopté par les producteurs hollandais, réduit les risques d'accroissement de populations dans les champs impliqués dans une rotation céréales/culture à forte valeur, comme la pomme de terre.

\section{Altises des tubercules}

L'altise des tubercules, Epitrix tuberis, cause des problèmes économiques impor- tants aux pommes de terre cultivées dans la région du nord-ouest de l'Amérique du Nord. Ce ravageur, que l'on croit originaire du Colorado (Etats-Unis), a été retrouvé en Californie, au Nebraska, au NouveauMexique, en Oregon, au Dakota du Sud, au Wyoming et dans l'état de Washington. Au Canada, sa distribution est restreinte à la Colombie-Britannique et à l'Alberta. L'espèce apparentée, E.cucumeris, est retrouvée dans toutes les provinces canadiennes et sur pratiquement tout le territoire continental des États-Unis. Les adultes d'E.tuberis et E.cucumeris se nourrissent du feuillage de la pomme de terre. Toutefois, seules les larves d'E. tuberis causent des dommages aux tubercules. Ces dommages consistent en des tunnels sur la surface des tubercules en croissance et des petits trous de $4-5 \mathrm{~mm}$ de profondeur dans les tissus des tubercules. La présence de tels dommages peut entraîner la déclassification de champs entiers ou le rejet des récoltes par les industries de transformation.

L'altise des tubercules présente deux générations par an en ColombieBritannique (Finlayson, 1950). En Colombie-Britannique, les adultes émergent à la mi-mai, trouvent une solanacée adaptée pour leur développement (préférentiellement la pomme de terre ou la tomate), se nourrissent et pondent des œufs pendant environ 40 jours. Les femelles pondent en moyenne 190 œufs en groupes sur le sol, près des plants. Les néonates se nourrissent en juin et en juillet des parties souterraines des plantes hôtes. Chez la pomme de terre, les dommages, qui consistent en des tunnels à la surface des tubercules, ont généralement peu d'impact économique, s'ils sont faits au cours de cette période. Les adultes de la génération d'été émergent à la mi-juillet et produisent une seconde génération de larves en août. Les populations d'E. tuberis sont alors les plus importantes de la saison: les dommages aux tubercules sont plus graves sur les cultivars tardifs. Les adultes de la seconde génération émergent en septembre et passent l'hiver dans le sol. Une seule femelle hibernante peut donner jusqu'à 20000 larves à la seconde génération, ce qui explique pourquoi des champs de pomme de terre, semblant exempts d'E. tuberis en début de saison, peuvent présenter des dommages importants à la récolte (Finlayson, 1950).

L'invasion d'un champ nouvellement établi par les adultes d'E. tuberis constitue une situation permettant une lutte aisée 
en périphérie des parcelles. En Colombie-Britannique, les consultants en lutte intégrée échantillonnent généralement des rangs périphériques de même que les rangs plus à l'intérieur des champs pour dépister les altises et autres insectes ravageurs. Afin de prévenir les pontes et le développement de générations ultérieures, Vernon et al., (1990) utilisent un seuil de 1 altise-adulte par 60 plants observés. Cette pratique a permis une réduction du nombre de traitements insecticides de huit traitements, sur toute la superficie du champ par an, à un seul traitement partiel (en périphérie des champs).

\section{Conclusion}

Comme la protection de la pomme de terre est un élément stratégique de cette culture importante au Canada, les producteurs embauchent du personnel qualifié pour dépister les insectes ravageurs présents dans leurs champs, déterminer leurs niveaux de populations et restreindre les traitements insecticides aux seules situations où les populations dépassent les seuils économiques. Ce choix repose sur des recherches entreprises par des organismes gouvernementaux, universitaires et privés. L'objectif commun des producteurs et des chercheurs est de développer et d'utiliser des stratégies antiparasitaires qui maintiennent les niveaux de production avec un minimum d'intrants agrochimiques.

\section{Références}

Boiteau G, Noronha C. Topical, residual and ovicidal contact toxicity of three reduced-risk insecticides against the European corn borer Ostrinia nubilalis (Lepidoptera: Crambidae) on potato. J Pest Man Sci 2007 ; 63 : 1230-8.

Boiteau G, Pelletier Y, Misener GC, et al. Development and evaluation of a plastic trench barrier for protection of potato from walking adult Colorado potato beetles (Coleoptera: Chrysomelidae). J Econ Entomol 1994 ; 87 : 1325-31.

Boiteau G, Vernon RS. Barrières physiques contre les insectes nuisibles. In : Vincent $C$, Panneton B, Fleurat-Lessard F, eds. La lutte physique en phytoprotection. Paris : Inra éditions, 2000.

Boiteau G. Recruitment by flight and walking in a one generation Colorado potato beetle (Coleoptera: Chrysomelidae) environment. Environ Entomol $2001 ; 30$ : 306-17.
Boiteau G. Timing of insecticide applications for the control of the Colorado potato beetle (Say) (Coleoptera: Chrysomelidae), on potatoes in New Brunswick. Can Entomol 1988 ; $120: 587-91$.

Boiteau G, Parry RH, Harris CR. Insecticide resistance in New Brunswick populations of the Colorado potato beetle (Coleoptera: Chrysomelidae). Can Entomol 1987 ; 119 : 459 63.

Boiteau G, Coleman W. Cold tolerance in the Colorado potato beetle Leptinotarsa decemlineata (say) (Coleoptera: Chrysomelidae). Can Entomol 1996 ; 128 : 1087-99.

Crozier S, Tanaka A, Vernon RS. Flight activity of Agriotes lineatus L. and A. obscurus L. (Coleoptera: Elateridae) in the field. J Entomol Soc Br Columbia $2003 ; 100$ : 91-2.

Doane JF, Lee YW, Klinger J, et al. The orientation response of Ctenicera destructor and other wireworms (Coleoptera: Elateridae) to germinating grain and carbon dioxide. Can Entomol $1975 ; 107: 1233-52$.

Dornan AP, Stewart JG. Population dynamics of the European corn borer Ostrinia nubilalis (Hübner) (Lepidoptera: Pyralidae) attacking potatoes in Prince Edward Island. Can Entomol 1995 ; 127 : 255-62.

Duchesne RM, Laguë C, Khelifi M, et al. Répression thermique du doryphore de la pomme de terre. In: Vincent C, Panneton B Fleurat-Lessard $\mathrm{F}$, eds. La lutte physique en phytoprotection. Paris : Inra éditions, 2000.

Eidt DC. European wireworms in Canada with particular reference to Nova Scotian infestations. Can Entomol 1953 ; 85 : 408-14.

Ericsson JD, Kabaluk JT, Goettel MS, et al. Spinosad interacts synergistically with the insect pathogen Metarhizium anisopliae against the exotic wireworms Agriotes lineatus and $A$. obscurus (Coleoptera: Elateridae). $J$ Econ Entomol $2007 ; 100: 31-8$.

Ester A, van Rozen K. Monitoring and control of Agriotes lineatus and $A$ obscurus in arable crops in the Netherlands. Insect pathogens and insect parasitic nematodes: Melolontha. IOBC WPRS Bull 2005 ; 28 : 81-6.

Finlayson DG. The effects of certain insecticides on the biotic potential of Epitrix tuberis Gentner (Coleoptera: Chrysomelidae). M.Sc. Thesis, University of British Columbia, Vancouver, B.C., 1950.

Glen R, King KM, Arnason AP. The identification of wireworms of economic importance in Canada. Can J Research 1943 ; 21 : 358-78.

Harris CR, Svec HJ. Colorado potato beetle resistance to carbofuran and several other insecticides in Quebec. J Econ Entomol 1981 ; $74: 421-4$.

Hoy CW, Wyman JA, Vaughn TT, et al. Food, ground cover, and Colorado potato beetle (Coleoptera: Chrysomelidae) dispersal in late summer. J Econ Entomol 1996 ; 89 : 963-9.

Inglis GD, Duke GM, Goettel MS, et al. Genetic diversity of Metarhizium anisopliae var. anisopliae in southwestern British Columbia. J Invertebr Pathol 2008 ; 98 : 101-13.

Kabaluk T, Goettel M, Erlandson M, et al. Metarhizium anisopliae as a biological control for wireworms and a report of some other endoparasitic enemies. Insect pathogens and insect parasitic nematodes: Melolontha. IOBC WPRS Bull $2005 ; 28$ : 109-16.
Kabaluk T, Vernon RS, Goettel M. Mortality and infection of wireworm, Agriotes obscurus (Coleoptera: Elateridae), with inundative field applications of Metarhizium anisopliae. Phytoprotection $2007 ; 88$ : 51-6.

Kennedy G, Anderson TE. European corn borer trapping in North Carolina with various sex pheromone component blends. J Econ Entomol $1980 ; 73: 642-6$.

Kennedy G. Effects of European corn borer (Lepidoptera: Pyralidae) damage on yield of spring-grown potatoes. J Econ Entomol 1983 ; spring-grown
$76: 316-22$.

Lee Jr. RE, Costanzo JP, Kaufman PE, et al. Icenucleating active bacteria reduce the cold hardiness of the freeze-tolerant Colorado potato beetle (Coleopetra: Chrysomelidae) J Econ Entomol $1994 ; 87: 377-81$.

Le Roux V, Brunissen L, Vincent C, Giordanengo $P$. Amélioration génétique de la pomme de terre et résistance aux pucerons : du terrain à la réponse moléculaire de la plante. Cah Agric $2008 ; 17: 401-6$

Minder IF. Hibernation conditions and survival rate of the Colorado potato beetle in different types of soil. In : Arnoldi KV, ed. Ecology and physiology of diapause in the Colorado beetle. New Delhi: Indian National Scientific Document Centre, 1976 ; (Reprinted by USDA.).

Nault BA, Kennedy GG. Sequential sampling plans for use in timing insecticide applications for control of European corn borer (Lepidoptera: Pyralidae) in potatoes. J Econ Entomol $1996 ; 89: 1468-76$.

Nault BA, Kennedy GG. Timing of insecticide applications for managing European corn borer (Lepidoptera: Pyralidae) infestations in potatoes. Crop Prot $1996 ; 15$ : 465-71.

Noronha C, Carragher D. Chemical control of the European corn borer on potatoes, 2006. Pestic Manage Res Rep 2006 ; 26 : 68-70.

Noronha C, Cloutier C. Effects of potato foliage age and temperature regime on prediapause Colorado potato beetle Leptinotarsa decemlineata (Coleoptera: Chrysomelidae) Environ Entomol 2007 ; 35 : 590-9.

Noronha C. Corn Borer Crusher: a device to control European corn borers in potatoes. AAFC-Crops and Livestock Research Centre Fact Sheet. 2006.

Noronha C, Cloutier C. Effects of soil conditions and body size on digging by prediapause Colorado potato beetles (Coleoptera: Chrysomelidae). Can J Zool 1998 ; 76 : 1705-13.

Noronha C, Cloutier C. Ground and aerial movement of adult Colorado potato beetle (Coleoptera: Chrysomelidae) in a univoltine population. Can Entomol 1999 ; 131 : 521-38.

Noronha C, Duke GM, Goettel MS. Damage potential and phenology of the Colorado potato beetle (Coleoptera: Chrysomelidae) on potato in southern Alberta. Phytoprotection $2002 ; 83: 89-98$.

Noronha C, Duke GM, Chinn JM, et al. Differential susceptibility to insecticides by Leptinotarsa decemlineata (Coleoptera: Chrysomelidae) populations from western Canada. Phytoprotection $2001 ; 82$ : 113-21.

Parker WE, Howard JJ. The biology and management of wireworms (Agriotes spp.) on potato with particular reference to the UK. Agric For Entomol $2001 ; 3$ : 85-98. 
Pelletier Y, Clark C. Use of reciprocal grafts to elucidate mode of resistance to Colorado potato beetle (Leptinotarsa decemlineata [Say]) and potato aphid (Macrosiphu $m$ euphorbiae [Thomas]) in six wild Solanum species. Am J Potato Res 2004 ; 81 . 341-6.

Pelletier Y, Clark C, Tai GCC. Resistance of three tuber-bearing potatoes to the Colorado potato beetle. Entomol Exp Applic $2001 ; 100$ 31-41.

Roush RT, Hoy CW, Ferro DN, et al. Insecticide resistance in the Colorado potato beetle (Coleoptera: Chrysomelidae): influence of crop rotation and insecticide use. J Econ Entomol $1990 ; 83: 315-9$.

Stewart JG. Monitoring adult European corn borer (Lepidoptera: Pyralidae) in potatoes on Prince Edward Island. Environ Entomol 1994 23 : 1124-8.

Stewart JG. The European corn borer, Ostrina nubilalis (Lepidoptera: Pyralidae): a potentia pest of potatoes grown on Prince Edward Island. Phytoprotection $1992 ; 73: 25-9$.

Stewart JG, Kennedy GG, Sturz AV. Incidence of insecticide resistance in populations of Colorado potato beetle, Leptinotarsa decemlineata (Say) [Coleoptera: Chrysomelidae], on Prince Edward Island. Can Entomol 1997 $129: 21-6$.

Toba HH, Pike KS, O'Keefe LE. Carbosulfan, fonofos, and lindane wheat seed treatments for control of sugarbeet wireworm. J Agric Entomol $1988 ; 5: 35-43$.
Ushatinskaya RS. Seasonal migration of adult Leptinotarsa decemlineata (Insecta, Coleoptera) in different types of soil and physiological variations of individuals in hibernating population. Pedobiologia (Jena) 1978 ; 18 : 120-6.

van Herk WG, Vernon RS. Effect of tempera ture and soil on the control of a wireworm Agriotes obscurus L. (Coleoptera: Elateridae) by flooding. Crop Prot 2006 ; 25 : 1057-61.

van Herk WG, Vernon RS. Soil bioassay for observing the orientation, feeding, repellency, and post-contact toxicity behaviours of wireworms (Coleoptera: Elateridae) exposed to insecticide treated wheat seed. Environ Entomol $2007 ; 36: 1441-9$

van Herk WG, Vernon RS, Tolman JH, et al. Mortality of a wireworm, Agriotes obscurus (Coleoptera: Elateridae) after topical application of various insecticides. J Econ Entomol $2008 ; 101: 375-83$.

Vernon RS, van Herk W, Tolman JH. European wireworms (Agriotes spp.) in North America: Distribution, damage, monitoring, and alternative integrated pest management strategies. Insect pathogens and insect parasitic gies. Insect pathogens and insect parasitic $2005 ; 28: 73-9$.

Vernon RS, LaGasa E, Philip H. Geographic and temporal distribution of Agriotes obscu rus and $A$. lineatus (Coleoptera: Elateridae) in British Columbia and Washington as determined by pheromone trap surveys. J Entomol Soc Br Columbia 2001 ; 98 : 257-65.
Vernon RS. Aggregation and mortality of Agriotes obscurus (Coleoptera: Elateridae) at insecticide-treated trap crops of wheat (Gramineae). J Econ Entomol 2005 ; 98 : 1999-2005.

Vernon RS, Mackenzie JR, Bartel DL. Monitoring tuber flea beetle, Epitrix tuberis Gentner (Coleoptera: Chrysomelidae) on potato: parameters affecting the accuracy of visual sampling. Can Entomol $1990 ; 122$ : 525-35.

Vernon RS, van Herk WG, Tolman JH, et al. Transitional sublethal and lethal effects of insecticides after dermal exposures to five economic species of wireworms (Coleoptera: Elateridae). J Econ Entomol 2008; 101 : 36574.

Vincent C, Boiteau G. La lutte contre les insectes nuisibles par aspiration. In : Vincent C, Panneton B, Fleurat-Lessard F, eds. La lutte physique en phytoprotection. Paris : Inra éditions, 2000.

Voss RH, Ferro DN. Phenology of flight and walking by Colorado potato beetle (Coleoptera: Chrysomelidae) adults in western Massachusetts. Environ Entomol 1990 ; 19 : 117-22.

Weber DC, Ferro DN. Distribution of overwintering Colorado potato beetle in and near Massachusetts potato fields. Entomol Exp Applic $1993 ; 66: 191-6$.

Wilkinson ATS. Wireworms of cultivated land in British Columbia. Proc Entomol Soc $\mathrm{Br}$ Columbia $1963 ; 60: 3-17$.

Wilkinson ATS, Finlayson DG, Morley HV. Toxic residues in soil 9 years after treatment with aldrin and heptachlor. Science $1964 ; 143$ : 681-2. 